\title{
ACUTE AORTIC DISSECTION WITH CRITICAL STENOSES OF THE TRUE LUMEN TREATED BY TRANSLUMINAL STENT-GRAFT PLACEMENT AND FINDINGS ONE YEAR AFTER TREATMENT
}

\author{
Takatsugu Shimono, MD, ${ }^{\mathrm{a}}$ Noriyuki Kato, MD, ${ }^{\mathrm{b}}$ Fuyuhiko Yasuda, MD, ${ }^{\mathrm{a}}$ Tadanori Hirano, MD, ${ }^{\mathrm{c}}$ Kan Takeda, MD, ${ }^{\mathrm{b}}$ and
} Iaso Yada, MD, ${ }^{\text {a }}$ Tsu and Matsuzaka, Japan

Stanford type B acute aortic dissection is mainly treated medically. However, emergency surgery for type B dissection with rupture, impending rupture, end-organ ischemia, or some combination thereof is mandatory. Surgical outcomes are poor for patients with an acute type B aortic dissection with endorgan ischemia. Recently, transluminal stent-graft placement became an alternative for the treatment of aortic dissection. ${ }^{1,2}$ However, transluminal stent-graft placement for stenosis of the true lumen is not common, and the fate of a narrowed true lumen is not clear after stent-graft treatment. We report the case of a patient with acute type B aortic dissection with critical stenoses of the true lumen treated by transluminal stentgraft placement. We also discuss the effects on a narrowed true lumen 1 year after the treatment.

From the Department of Thoracic and Cardiovascular Surgery, ${ }^{a}$ the Department of Radiology, ${ }^{\mathrm{b}}$ Mie University School of Medicine, Tsu, and the Department of Radiology, ${ }^{c}$ Matsuzaka Chu-o General Hospital, Matsuzaka, Japan.

J Thorac Cardiovasc Surg 2001;121:989-92

Copyright (C) 2001 by The American Association for Thoracic Surgery

$0022-5223 / 2001 \$ 35.00+0 \quad \mathbf{1 2 / 5 4 / 1 1 0 4 8 6}$

doi: $10.1067 / \mathrm{mtc} .2001 .110486$
Clinical summary. A 54-year-old woman with a sudden onset of back pain was admitted to a satellite hospital of Mie University. Contrast-enhanced computed tomography (CT) revealed a DeBakey type IIIb, Stanford type B acute aortic dissection. The patient was treated medically. Nineteen days after admission, she had acute renal failure (blood urea nitrogen, $54 \mathrm{mg} / \mathrm{dL}$; creatinine, $3.8 \mathrm{mg} / \mathrm{dL}$ ) and left leg ischemia. CT disclosed severe stenosis of the true lumen at the level of the left renal artery. She was referred to Mie University Hospital for evaluation and treatment of acute aortic dissection. Her left femoral pulse was not palpable. The patient immediately underwent digital subtraction angiography (DSA), which showed that the intimal tear was located in the midportion of the descending thoracic aorta and that stenoses of the true lumen were located at the level of the diaphragm and distal to the level of the left renal artery. The left external iliac artery also had a critical stenosis. The true lumen of the right iliac artery was completely obliterated. However, the right leg was perfused by blood flow through the false lumen (Fig 1, A).

Emergency transluminal stent-graft placement was subsequently performed. The stent graft was constructed of a selfexpanding, Gianturco stainless steel Z stent (Cook Critical Care, Inc, Bloomington, Ind) covered with a balloon-expand- 

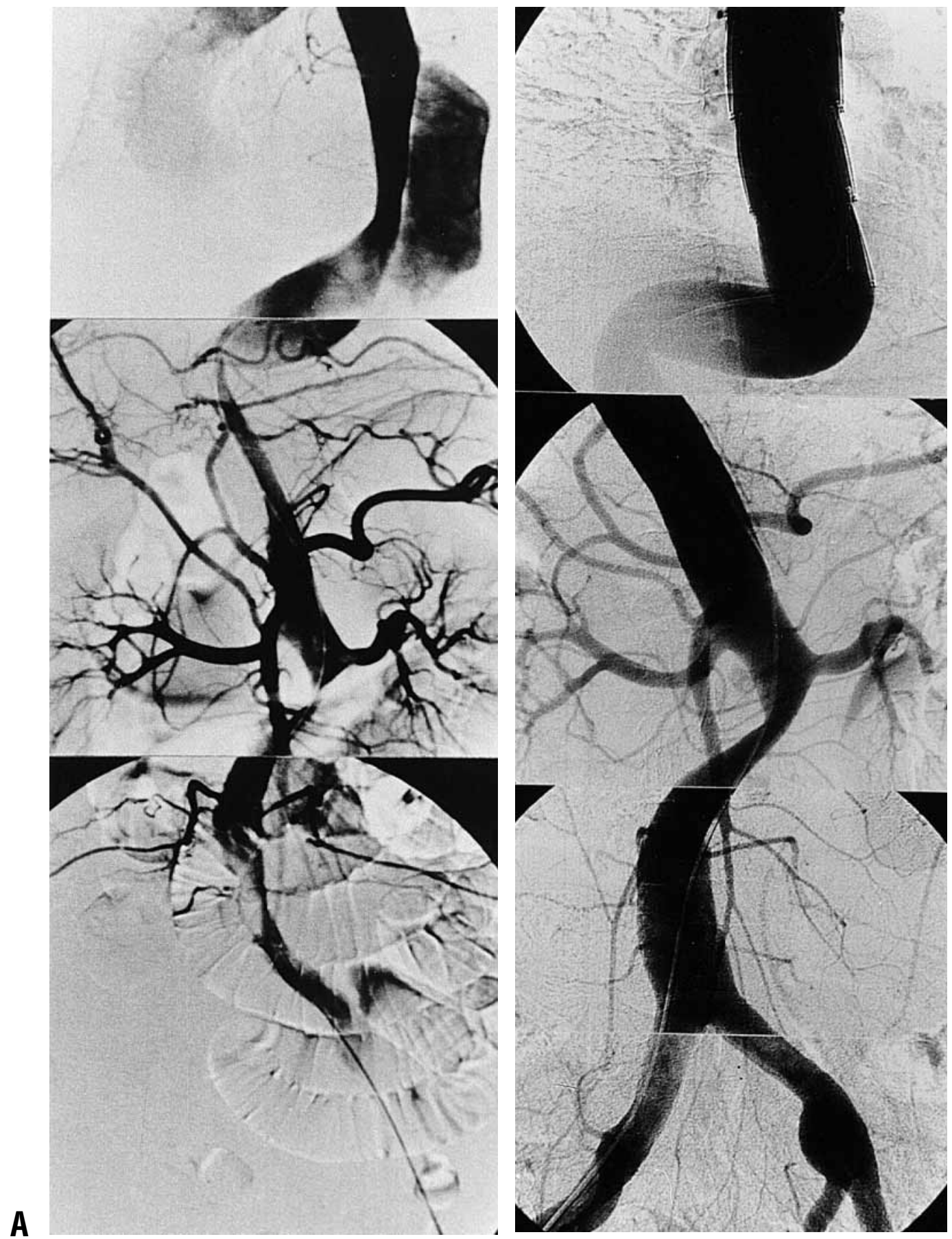

Fig 1. A, DSA before the stent-graft placement showing the intimal tear located in the midportion of the descending thoracic aorta, critical stenoses of the true lumen, and severe narrowing of the left external iliac artery. B, DSA 2 weeks after the repair showing no evidence of tear in the thoracic aorta, no endoleak at the site of the stent graft, and stenosis just distal to the left renal artery.

ed polytetrafluoroethylene graft (Bard, Inc, Tempe, Ariz). The outer diameter of the stent graft was $28 \mathrm{~mm}$, and the length of the stent graft was $75 \mathrm{~mm}$. The stent graft was placed in the middle portion of the descending thoracic aorta through the left common femoral artery after achievement of general anesthesia. DSA was performed after stent-graft placement and showed that the intimal tear was completely excluded by the stent graft. The patient's renal function improved, and her left femoral pulse was palpable after the treatment. 

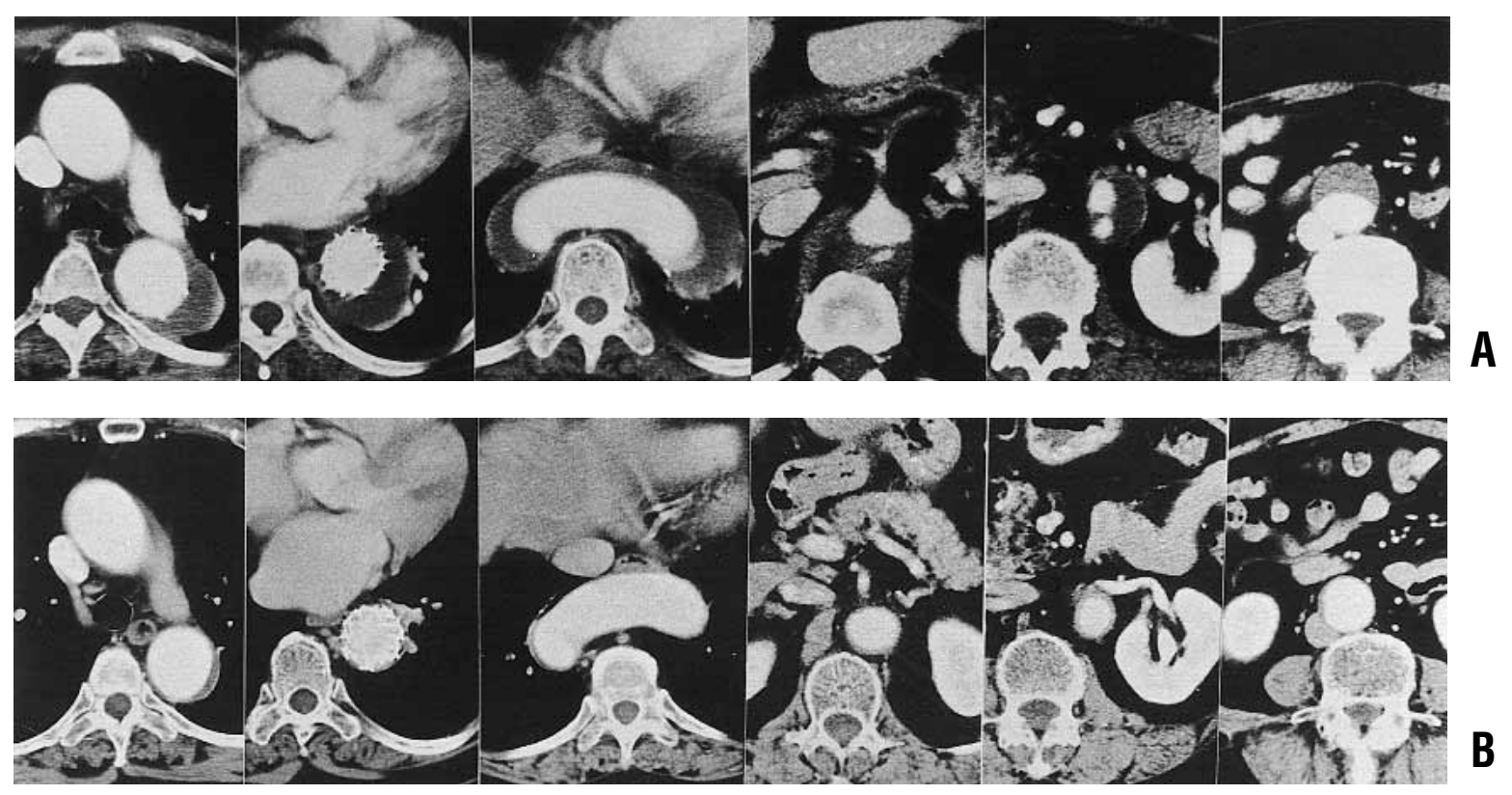

Fig 2. A, Contrast-enhanced CT 4 weeks after stent-graft placement showing thrombosis of the false lumen in the descending thoracic aorta and remaining narrowing of the true lumen just distal to the left renal artery. B, Contrastenhanced CT 1 year after stent grafting showing elimination of the false lumen and complete enlargement of the true lumen to its normal size.

DSA performed 2 weeks after the repair confirmed that there was no intimal tear in the entire thoracic aorta and no endoleak. The true lumen had enlarged, and stenoses at the level of the diaphragm and in the left iliac artery were improved. However, moderate stenosis just distal to the left renal artery still remained (Fig 1, B). Four weeks after stentgraft placement, contrast-enhanced CT revealed that the false lumen in the descending thoracic aorta had completely thrombosed and that the true lumen had enlarged. However, the true lumen just distal to the left renal artery was still narrowed (Fig 2, A). Contrast-enhanced CT performed 1 year after stent grafting revealed elimination of the false lumen from the level of the descending aorta to the level of the aortic bifurcation. CT also showed that the true lumen had enlarged to its normal size. The narrowing distal to the left renal artery had also resolved (Fig 2, B). The patient is alive and well 2 years after the endovascular stent-graft repair. Her current renal function is normal (blood urea nitrogen, 9 $\mathrm{mg} / \mathrm{dL}$; creatinine, $0.8 \mathrm{mg} / \mathrm{dL}$ ). Also, her pulsations of posterior tibial arteries in both legs are good, and she shows no intermittent claudication.

Discussion. Medical treatment for Stanford type B acute aortic dissection represents the first-line therapy, and its results are better than those of surgical treatment. However, the existence of critical complications, such as rupture, impending rupture, and end-organ ischemia, leads to emergency operations, the outcome of which are still poor. ${ }^{3}$ In 1994, Dake and coworkers ${ }^{4}$ reported the feasibility of endovascular stent grafting for descending thoracic aneurysms. Recently, Stanford University and Mie University groups reported the feasibility of endovascular stent grafting for acute aortic dissection. ${ }^{1}$ German and Italian groups have also reported that the early results of endovascular stent-graft repair are better than those of conventional surgical treatment for chronic type B aortic dissection. ${ }^{2}$ Some institutions already started clinical trials of endovascular stent-graft repair for acute aortic dissection. However, this treatment for critical stenosis or obliteration of the true lumen with end-organ ischemia is not common. In addition, the fate of a critically narrowed true lumen after stent-graft treatment is not clear.

Slonim and coworkers ${ }^{5}$ reported on other alternatives for true lumen obliteration: percutaneous balloon fenestration and stenting. ${ }^{5}$ In our experience most cases of this subtype of dissection do not have major reentry. This means that remodeling of the aortic lumen could continue, the true lumen could be completely enlarged, and the false lumen could disappear, even if the true lumen is still narrow immediately after treatment. Therefore, theoretically, stent-graft placement to exclude the primary entry site should be the first choice of treatment for this subtype of acute aortic dissection. In our case, stenosis of the true lumen and a patent false lumen existed 1 month after stent-graft placement, but the aortic lumen was completely recovered after 1 year.

In conclusion, it appears that endovascular stent grafting was a good therapeutic option in this patient with acute type B dissection with true lumen stenosis and end-organ ischemia, particularly in light of the difficulty of the surgical approach. 
Received for publication June 29, 2000; accepted for publication July 28, 2000.

Address for reprints: Takatsugu Shimono, MD, Department of Thoracic and Cardiovascular Surgery, Mie University, School of Medicine, 2-174 Edobashi, Tsu, Mie 514, Japan (E-mail: simono-t@ @ clin.medic.mie-u.ac.jp).

\section{REFERENCES}

1. Dake MD, Kato N, Mitchell RS, et al. Endovascular stent-graft placement for the treatment of acute aortic dissection. N Engl J Med 1999;340:1546-52.
2. Nienaber CA, Fattori R, Lund G, et al. Nonsurgical reconstruction of thoracic aortic dissection by stent-graft placement. N Engl J Med 1999;340:1539-45.

3. Miller DC. The continuing dilemma concerning medical versus surgical management of patients with acute type B dissection. Semin Thorac Cardiovasc Surg 1993;5:33-46.

4. Dake MD, Miller DC, Semba CP, Mitchell RS, Walker PJ, Liddell RP. Transluminal placement of endovascular stent-grafts for the treatment of descending thoracic aortic aneurysms. N Engl J Med 1994;331:1729-34.

5. Slonim SM, Miller DC, Mitchell RS, Semba CP, Rasavi MK, Dake MD. Percutaneous balloon fenestration and stenting for life-threatening ischemic complications in patients with acute aortic dissection. J Thorac Cardiovasc Surg 1999;117:1118-26. 